\title{
Difficult-to-Treat-Depression and GPs' Role: Perceptions of Psychologists
}

\author{
Kay M. Jones*, Leon Piterman \\ Office of the Pro Vice-Chancellor, Peninsula Campus, Monash University, Frankston, Australia \\ Email: ${ }^{*}$ kay.jones@monash.edu
}

Received 17 October 2014; revised 13 November 2014; accepted 10 December 2014

Academic Editor: Gjumrakch Aliev, University of Atlanta, USA

Copyright (C) 2015 by authors and Scientific Research Publishing Inc.

This work is licensed under the Creative Commons Attribution International License (CC BY). http://creativecommons.org/licenses/by/4.0/

(c) (i) Open Access

\begin{abstract}
Introduction: Since the Australian Government introduced the "Better Access to Mental Health Services" program in 2006, psychiatrists, psychologists and general practitioners (GPs) have become increasingly involved in service provision for people seeking help with mental health problems. The aim of this research was to a) explore psychologists' perceptions of difficult to treat depression (DTTD) and b) explore what they thought about the GPs' role in managing these patient given that most patients are referred to psychologist by GPs. Methods: A previously developed semi-structured interview schedule comprising six questions was used. Seven psychologists participated in a focus group held in Melbourne. Data were analysed using the framework method. Findings, including Discussion: While psychologists understood the term DTTD it was suggested that using different terms may limit understanding between health professionals. Rather than diagnosing, psychologists were more likely to conduct further assessment contextually to confirm GPs' diagnosis. Communication with GPs was important, particularly when managing "long-term" and suicidal patients. Management included cognitive and behavioural interventions and referring to other mental health services, psychiatrists and/or other allied health professionals. Referral to psychiatrists could be difficult because of limited availability and for some patients, prohibitive costs. Although psychologists discussed non-pharmacological and/or complementary treatment options with patients, they were more likely to rely on GPs to discuss/prescribe these options. Conclusion: While generalisability may be limited, this study is the first to document some understanding of psychologists perceptions of DTTD and the importance of GPs and other health professionals' role in managing this patient cohort.
\end{abstract}

\section{Keywords}

GPs' Role, Difficult-to-Treat-Depression

\footnotetext{
${ }^{*}$ Corresponding author.
} 


\section{Introduction}

Mental health and mental illnesses are determined by multiple and interacting social, psychological and biological factors [1]. Since 1992, the Australian Government has introduced several initiatives focussing on mental health [2] [3], including the "Better Access to Mental Health Services Psychiatrists, Psychologists and General Practitioners" (including the access to allied psychological services (ATAPS) component [4] [5]) through the Australian government Medicare benefits schedule (MBS) initiative in November 2006 [6]-[8]. During the first full year of better access (2006-2007), expenditure was \$583 million; by 2010-2011, the overall MBS mental health specific expenditure figure rose to $\$ 852$ million, accounting for $35 \%$ of overall Australian government mental health spending, and during that period, the percentage of the population receiving MBS funded services rose from $3.1 \%$ to $6.9 \%$ [2] [9].

These reforms resulted in a change in service provision with a greater emphasis on care within the community rather than institutions and/or hospitals [10]. In 1992-1993, services provided by psychiatrists and general practitioners (GPs) accounted for all of the Australian government funded MBS expenditure on mental health services [2]. By 2011-2012, psychologists were providing the largest proportion of MBS subsidised services (43.5\%); GPs were providing $27.7 \%$ of the services, which were mainly for the provision of GP mental health treatment items [11].

In Australia, for the majority of people seeking help with mental health problems, the first point of contact is the GP [12]. In 2011-2012, an estimated 12.1\% of GP encounters were mental health-related and of these, over one third (34\%) were for depression - the most commonly managed problem by a GP in a mental health-related encounter [13]. Thus GPs are heavily involved in managing patients diagnosed with depression [3].

While evidence suggests that the uptake of the better access initiative has been high [3] and there has been an increased appreciation by health professionals of each other's role [6] [14], access to psychological services remains an issue, particularly in the private sector where services are often beyond the financial reach of many patients despite the Medicare subsidy [12]. Also noted as a concern was communication between health professionals; some GPs felt that communication was not always optimal, indicating they felt they don't receive sufficient feedback about patients' progress [6].

In 2011-2012, there were an estimated 1858 (full-time-equivalent) registered psychologists practising [15] in Australian states and territories. Specialisations in psychology include clinical, community, counselling, educational and development, organisational, neuropsychology and health psychology; all require additional postgraduate study and training [12] [16]. The public sector is a major employer of psychologists who consult with individuals and groups, assess psychological disorders, and administer treatment programs such as focussed psychological strategies and psychological therapy services [11] [12].

A search of the literature found a study published in 2004 describing the benefits of a collaborative model of mental health care involving GPs and clinical psychologists for patients with common mental disorders who sought help via primary care health providers [17]. Another, published in 2012 compared the better access initiative with the better outcomes in mental health care initiative and reported an enormous increase in access to primary care management of depression, although advantaged major city patients gained most [3]. Similarly, Australian government publications describe outcomes of the various initiatives; a report published in 2011 evaluating the better access initiative found, among other things that outcome and cost for consumers seen by psychologists suggested that the initiative was providing good value for money [6]. The National Mental Health Report 2013 described various aspects of the progress of mental health reforms in Australia including the doubling of funding in the 2011-2012 federal budget for the ATAPS [4] [5] component of the better access initiative [2] [8]. But, no literature could be found that described psychologist's perceptions and/or experiences of managing patients diagnosed with difficult-to-treat depression (DTTD) or their relationship with GPs who referred these patients to them.

Thus the absence of literature suggests the importance of conducting exploratory work to gain an understanding of psychologists' perceptions, and at the same time, adding to the exploration of GPs' [18], GP trainees' [19] and psychiatry registrars' [20] experiences as previously described by the authors.

The aims of this research were a) to explore psychologists' perceptions of DTTD and b) to explore what they think about the GPs' role in managing patients diagnosed with DTTD.

\section{Methods}

As the DSMV [21] does not provide a clear definition for diagnosing and/or managing DTTD, this paper uses 
the description of DTTD as "most often conceptualized in terms of repeated failures to ameliorate depressive symptoms" [22].

\subsection{Sample Recruitment}

A convenience sample was recruited via an email forwarded to psychologists with links to the Monash Medical Centre, a public hospital in Melbourne, Australia. When potential participants responded and agreed to participate, they provided their contact details (email) to the research team for the purpose of the research team advising time, date and venue for the focus group [23] [24].

\subsection{Data Collection and Analysis}

A semi-structured interview schedule comprising six headings was used; this schedule was previously developed and used when interviewing and/or conducting focus groups with GPs [18], GP trainees [19] and psychiatry registrars [20] (Table 1$)$.

All data were collected in Melbourne; a focus group was held with seven psychologists (five females, two males) which lasted approximately one-and-a-half-hours, was audio-taped and transcribed verbatim. At the time of the focus group, all described themselves as clinical psychologists with experience in both the public (including hospitals) and private sector. No other demographic data were collected.

Data were analysed using the framework method [26] to understand participants' perspectives. Data were analysed manually and independently by the investigators. When there was a difference of opinion, the issues were discussed and agreement reached [26]. Findings, including discussion are reported under the interview schedule's six headings. Comments are reported as P1-P7.

Ethics approval to conduct the study was obtained from Monash University Human Research Ethics Committee (MUHREC).

\section{Findings and Discussion}

Findings and discussion are reported under the interview schedule's six sub-headings.

Question 1: Understanding of the term difficult-to-treat depression (DTTD).

Whilst GPs' understanding of the term DTTD varied [18], all psychologists had a clear understanding of DTTD, describing DTTD as certainly more than uncomplicated depression, but may be difficult to treat for a range of reasons including that health professionals may not be able to engage the person in any level of responsiveness in self-management, and/or there may be co-morbid problems. In addition, it was suggested that terms other than DTTD may be used by health professionals to describe a particular patient's diagnosis, thus there may not be clear understanding between health professionals of the meaning of DTTD. One participant suggested that to understand the term, the patient and/or the problem:

\section{Table 1. Interview schedule.}

1. What is your understanding of the term difficult-to-treat-depression (DTTD)?

2. What is your understanding of other terms; viz: treatment-resistant depression (TResD) treatment-refractory depression (TRefD), treatment-resistant major depressive disorder (TRMDD) and major depressive disorder (MDD)?

3. What are your experiences of diagnosing DTTD?

4. What are your experiences of managing DTTD?

5. Does your management of these patients include:

a. using an illness management model or a chronic illness model [25];

b. communication with GPs;

c. referring the patient to other allied health professionals; and

d. suggesting/prescribing other non-pharmacological and/or complementary treatment?

6. If “no” to any of Question 5, have you ever considered using any of these options/other comments? 
We start with an assessment, actually working out what were the presenting problems. (P1)

I think there's also an element that you aren't able to engage the person in any level of responsiveness in self-management or anything like that, minimum insight. (P7)

Question 2: What is your understanding of other terms viz: treatment-resistant depression, treatmentrefractory depression, treatment-resistant major depressive disorder, and major depressive disorder (MDD)?

There was agreement that the terms could be used inter-changeably, thus may have different meanings for health professionals and/or patients. It was also suggested that different terms may be tied to medical expectation and/or medication (P7):

If the GP has tried different medication from their viewpoint, the patient may be treatment resistant, but then we look at the psychological side, this is where the psychologist could be certainly enhancing the biological side. (P2)

There could also be other co-morbid problems that are going as well, personality traits, abuse, family issues. (P5)

The variance of psychologists' understanding of the terms when compared to the GPs' [18] and GP trainees' [19] understanding, may reflect the difference in emphasis of the professional roles and training.

Question 3: What are your experiences of diagnosing DTTD?

Whilst all GPs had diagnosed patients with DTTD, similar to GP trainees and psychiatrist registrars, psychologists' experiences varied. The majority of psychologists agreed that if a client was referred with a diagnosis of DTTD, then they would not spend time confirming the diagnosis or otherwise (P3), rather, would do a further assessment contextually. One suggested starting a discussion about personality issues to check if that was the overriding or prime problem and that their [patient's] low mood was sort of secondary to a different problem rather than being the primary issue (P1). Another suggestion was that if they [the psychologists] were unsure of the diagnosis as indicated in the referral provided, they would take the query back to the referring GP, particularly to check whether different medication had been tried (P3).

Whilst there was agreement that a change in medication may be helpful for some patients because some respond to some medications and not to others (P1), no-one would change medication, rather, the matter would be referred back to the referring GP:

My impression is that where a psychologist can particularly add value is getting the persons perspective of what's going on, not just the illness, how they actually live it, experience it and how it impacts on their daily level of functioning would be of particular interest. (P2)

Is the GP monitoring medication, risk, and has suicidal ideation increased as a result of being on medication? (P4)

The last comment moved the discussion from medication changes to unanticipated suicide. Whilst all had heard of another health professional having to deal with a suicide, no one in this group had that experience, but two had dealt with uncompleted attempts (P1, P2). In addition to dealing with uncompleted attempts, several GPs had dealt with completed suicide [18]. Concern was expressed regarding whether the GP was monitoring risk (P6) and whether any subsequent emerging legal issues may emerge, particularly for the health professionals involved with the client.

Question 4: What are your experiences of managing DTTD?

All started managing the patient with an assessment to work out what the presenting problems were by looking at the psychological aspects. In a hospital, the psychologist tends to use more of a clinical interview rather than a checklist, but when there is a workcover claim, or the patient is in the private health system, there are requirements for the psychologist to use outcome measures such as K10 or BECK [11] [12] and document outcome measures at timelines (P3, P5, P7). The process includes focussing on self-care quality of life aspects and working in the framework of negotiating tasks, monitoring how the patient is progressing, utilising mood boosting strategies, discussing the role of exercise, sleep and the social context (P2). Management includes using CBT-based cognitive and behavioural interventions, psycho-education and/or interpersonal therapy [4] [5]. All 
agreed that the meaning and purpose of life changed with age, gender and cultural expectations.

As part of management, psychologists and GPs [18] commented on the difficulty when endeavouring to involve other services in managing patients with chronic depression that isn't changing. Various reasons were mentioned including that these patients don't meet the threshold for adult mental health services (P6) and that endeavours to find private psychiatrists are often limited by the financial concerns of the patient [19]. All agreed there is a proportion of patients who can't afford services provided by psychologists and psychiatrists when bulk-billing is not available [12]. As at March 2012, Medicare rebates were available for maximum of ten visits per calendar year and an additional six mental health services under "exceptional circumstances" [7]:

In primary health there's always a query around bulk billing psychologists and psychiatrists—there's a financial cost that a proportion of people just can't afford. (P3)

There's a lot of factors that keep them unwell because that's how they see themselves, there's an unconscious motivation to stay well, if that makes sense. (P1)

I have some experience where the client was referred to other services and it wasn't necessarily good outcome for the client; that's very disappointing as well. (P7)

Question 5: Does your management of these patients include: a) using an illness management model [25]; b) communication with GPs; c) referring to allied health professionals, and/or; d) suggesting/prescribing non-pharmacological and/or complementary treatment?

a) Using an illness management model

Along with GP trainees [19] and psychiatry registrars [20], these psychologists had not heard of the Wagner illness model [25]. However, the psychologists had heard of and used various tools including the K10 and BECK, and a range of interventions including CBT-based cognitive and behavioural interventions, psychoeducation, and interpersonal therapy to reduce distress and enhance and promote emotional wellbeing [4] [5] [8].

b) Communication with GPs

General consensus was that, generally, people are now more willing to acknowledge and address mental health issues and seek help, initially from GPs. All participants had communicated with GPs and agreed with the GPs comments that navigating the various aspects of the mental health system could be difficult. This sometimes resulted in GPs using mental health treatment items [11] to refer patients to psychologists and/or other primary mental health services [18]. Psychologists found that, for them, referring an adult to primary mental health services can mean being on the phone for quite some time (P1); thus it can be tricky for GPs because they have so little time (P5). Community health was described as a "last remaining resort" for patients who have gone through the whole system, have seen many GPs and may end up with "a concoction of stuff”, and no one is sufficiently knowledgeable about those patients (P7):

I seriously think, as people working within the system, navigating the system is incredibly difficult, particularly for someone not functioning; they need as much help as they can get. (P5)

I tend to write to the GP to refer in a specific way because that's what they need to access the service. (P6)

Sometimes GP's won't know any more than you about the system, or you would know more about mental health services. (P4)

Some GPs I know find it increasingly hard to manage people who are very chronically mentally unwell because they don't have the time to sit down and do more counseling based sessions. (P1)

One psychologist had worked in the country in a large rural centre and like the GPs who worked in rural Australia, found it "tough" to get services, albeit mental health support is more accessible in recent years because of the Medicare rebates and psychologists and social workers working in the community (P4).

c) Referring to other allied health professionals

Referring to other allied health professionals was limited, but it was generally agreed that it was difficult when referring to a multi-disciplinary team because "no one works on the same day" (P1):

If you're in the hospital system you can probably run by the GP, but if you need someone who's more down the psychiatry line, you can't get everyone together in the same way. If you need the psychiatrist, the GP and psychologist together you rarely can do that. (P5) 
Challenges were also experienced when endeavouring to deal with a crisis using the Crisis Assessment Team (CAT):

I've had people swearing that you can't get the CAT team to go and see somebody or make phone calls. (P2)

The CAT team goes out in the area where I work until $10 \mathrm{pm}$, and then people go to the emergency department of the local hospital for the night shift workers to assess. (P3)

Whilst referral patterns differed between groups, general consensus was that, regardless of availability of resources or cost, the relationship between the patient and professional was particularly important [18]-[20].

d) Suggesting/prescribing non-pharmacological and/or complementary treatment

According to the Australian Psychological Society, psychologists spearheaded the development/use of nonpharmacological treatments [12]. Around half of the cohort had recommended exercise including yoga and Pilates. All agreed they would ask about these alternate options such as St John's Wort during the assessment process because of significant interactions with drugs:

We hope that they've [patients] discussed that with the GP or the GP has discussed it. (P5)

They [patients] don't always talk about it because they don't consider it being medication. (P6)

Similarly, GPs felt that non-pharmacological, complementary and/or lifestyle options have a role in managing DTTD [18] regardless of whether the GP suggested, or patients independently explored options [19].

Question 6: If no to any of Question 5, have you ever considered using any of those options/other comments?

The discussion concluded by returning to the importance of "knowing the system" and any subsequent impact on health professionals and patients: most participants felt the referral process is difficult and "you have to be pretty assertive and then that can be misinterpreted" (P3). Some services have criteria which are understandable, but there is also the stigmatising issue:

Stigmatizing is still a problem, possibly in response to the psychotic patient; it's about containment whether in the community in-patient unit and medication, and requiring more time than is available. (P6)

Making referrals for someone with difficult to treat depression, was described as more difficult if the person's not particularly receptive to accepting service:

That still happens; it's nothing to do with need. (P6)

\section{Conclusions}

This paper is the first to contribute to understanding psychologists' perceptions of DTTD and at the same time, adding to the exploration of GPs [18], GP trainees [19] and psychiatry registrars' [20] experiences.

These psychologists demonstrated a clear understanding of DTTD, insight into various terms and their professional role and relationship with GPs in diagnosing and managing patients diagnosed with DTTD. Although the Australian government's initiatives had improved access to psychologists, particularly via GP referrals, concerns were expressed about the difficulty GPs and psychologists experienced when endeavouring to access services and also the cost of services for those who were unable to access services via Medicare funding options.

Opinion on communication varied; while referrals were made by GPs to psychologists as part of the diagnosis and management process, these referrals could be hampered initially by limited access/availability of psychologists and other health professionals, then, during the management process when these health professionals endeavoured to hold multi-disciplinary team discussions. After-hours services such as Crisis Assessment Teams were also described as difficult to access, resulting in people who were mentally unwell having to attend already overloaded emergency department in local hospitals.

While the generalisability of this study may be limited because of the small number of participants who were all from metropolitan Melbourne, this study is the first to contribute to the literature about psychologists' experiences and perceptions of DTTD, what psychologists think about the GPs' role in managing patients with this 
diagnosis and drawing some comparisons between the various health professionals' experience in diagnosing and managing with DTTD.

\section{References}

[1] Australian Bureau of Statistics (2007) National Survey of Mental Health and Wellbeing: Summary of Results (4326.0). www.abs.gov.au

[2] Australian Government Department of Health and Ageing (2013) National Mental Health Report 2013: Tracking Progress of Mental Health Reform in Australia 1993-2011. Commonwealth of Australia, Canberra.

[3] Harrison, C.M., Britt, H.C. and Charles, J. (2012) Better Outcomes or Better Access—Which Was Better for Mental Health Care? MJA, 197, 170-172.

[4] Australian Government Department of Health (2014) Introduction, Policy Imperatives, Background. http://www.health.gov.au/internet/publications/publishing.nsf/Content/mental-boimhc-ataps-review-toc $\sim$ mental-boimh c-ataps-review-appb mental-boimhc-ataps-review-appb-int

[5] Fletcher, J., King, K., Bassilios, B., Reifels, L., Blashki, G., Burgess, P. and Pirkis, J. (2012) Evaluating the Access to Allied Psychological Services (ATAPS) Program. Centre for Health Policy Programs and Economics, University of Melbourne, Melbourne. https://ataps-mds.com/site/assets/files/1019/19th_interim_evaluation_report.pdf

[6] Pirkis, J., Harris, M., Hall, W. and Ftanou, M. (2011) Evaluation of the Better Access to Psychiatrists, Psychologists and General Practitioners through the Medicare Benefits Schedule Initiative. Summative Evaluation, Final Report. Centre for Health Policy, Programs and Economics, University of Melbourne, Melbourne.

[7] Australian Government Department of Health and Ageing (2012) Fact Sheet. New Transition Arrangements for the Allied Mental Health Services Available under the Better Access Initiative. www.health.gov.au/internet/main/publishing.nsf/Content/16AB49E865EF306DCA257BF0001BDA53/\$File/transfac.p $\underline{\mathrm{df}}$

[8] Australian Psychological Society (2013) APS Fact Sheet. Medicare Rebates for Mental Health Services Provided by Psychologists. www.psychology.org.au/publications/inpsych/gps/

[9] Reavley, N.J. and Jorm, A.F. (2014) Mental Health Reform: Increased Resources but Limited Gains. MJA, 201, 375376.

[10] Private Mental Health Alliance (PMHA) (2011) Principles for Collaboration, Communication and Cooperation between Private Mental Health Service Providers. Private Mental Health Alliance, Kingston ACT.

[11] AIHW (2013) Mental Health Services-In Brief 2013. AIHW, Canberra.

[12] Senate Select Committee (2006) Mental Health: A National Approach to Mental Health—From Crisis to Community. Final Report, Senate Select Committee, Commonwealth of Australia, Canberra.

[13] AIHW (2013) Mental Health-Related Services Provided by General Practitioners. https://mhsa.aihw.gov.au/services/general-practice/

[14] Wyman, K. and Stokes, D. (2014) Psychologists and GPs: How to Find Common Ground. Australian Psychological Society. https://mhsa.aihw.gov.au/services/general-practice/

[15] AIHW (2012) Mental Health Services in Australia-Staffing. https://mhsa.aihw.gov.au/resources/facilities/staffing/

[16] Australian Psychological Society (2014) Study Pathways. www.psychology.org.au/studentHQ/studying/study-pathways/

[17] Vines, R., Richards, J.C., Thomson, D.M., Brechman-Toussaint, M., Kluin, M. and Vesley, L. (2004) Clinical Psychology in General Practice: A Cohort Study. The Medical Journal of Australia, 181, 74-77.

[18] Jones, K.M., Castle, D.J. and Piterman, L. (2012) Difficult-to-Treat-Depression: What Do General Practitioners Think? The Medical Journal of Australia, 1, 6-8.

[19] Jones, K.M., Piterman, L. and Spike, N. (2014) Difficult-to-Treat-Depression—Perceptions of GPs and GP Trainees. Open Journal of Psychiatry, 4, 228-237.

[20] Jones, K.M. and Piterman, L. (2014) Difficult-to-Treat-Depression and GPs’ Role: Perceptions of Psychiatry Registrars. Open Journal of Psychiatry, 4, 301-308.

[21] American Psychiatric Association (2013) DSMV. 5th Edition, American Psychiatric Publishing, Washington DC.

[22] Grote, N.K. and Frank, E. (2003) Difficult-to-Treat Depression: The Role of Contexts and Comorbidities. Society of Biological Psychiatry, 53, 660-670. http://dx.doi.org/10.1016/S0006-3223(03)00006-4

[23] Liamputtong, P. and Ezzey, D. (2005) Qualitative Research Methods. Oxford University Press, Melbourne.

[24] Polgar, S. and Thomas, S. (2005) Introduction to Research in the Health Sciences. Elsevier Churchill Livingstone, 
Sydney.

[25] Wagner, E.H. (1998) Chronic Disease Management: What Will It Take to Improve Care for Chronic Illness? Effective Clinical Practice, 1, 2-4.

[26] Ritchie, J. and Spencer, L. (1994) Qualitative Data Analysis for Applied Policy Research. In: Bryman, A. and Burgess, B., Eds., Analysing Qualitative Data, Routledge, London. http://dx.doi.org/10.4324/9780203413081_chapter_9 
Scientific Research Publishing (SCIRP) is one of the largest Open Access journal publishers. It is currently publishing more than 200 open access, online, peer-reviewed journals covering a wide range of academic disciplines. SCIRP serves the worldwide academic communities and contributes to the progress and application of science with its publication.

Other selected journals from SCIRP are listed as below. Submit your manuscript to us via either submit@scirp.org or Online Submission Portal.
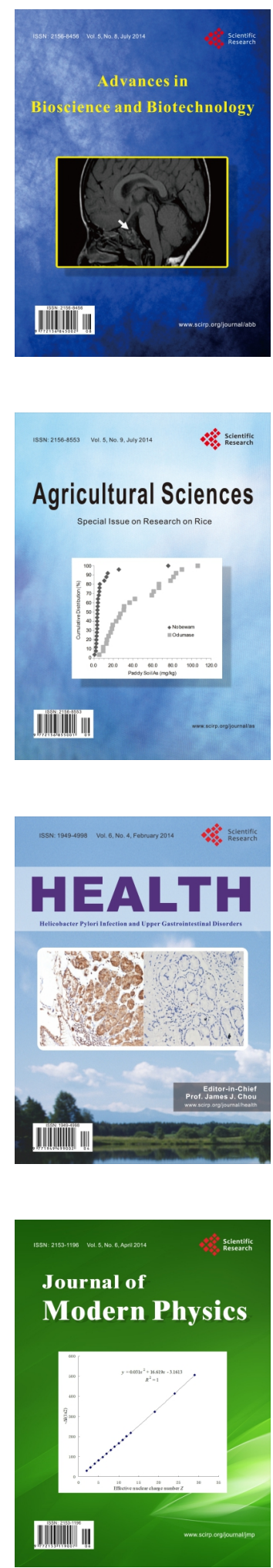
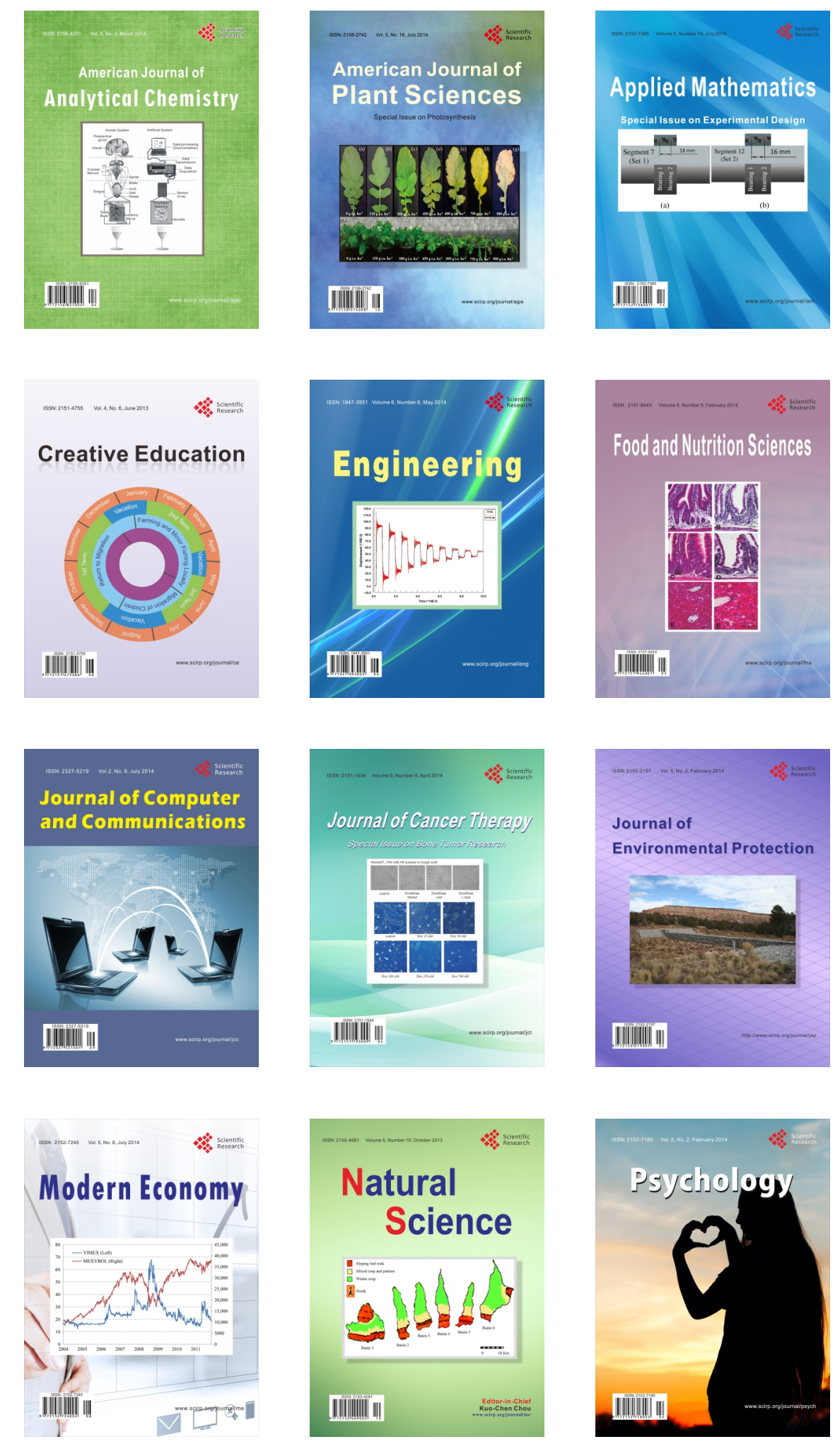